Revista Brasileira de Agricultura Irrigada v.7, nº 1, p. 27 - 41, 2013

ISSN 1982-7679 (On-line)

Fortaleza, CE, INOVAGRI - http://www.inovagri.org.br

DOI: $10.7127 /$ rbai.v7n100101

Protocolo 101.12 - 15/10/2012 Aprovado em 06/03/2013

\title{
DESEMPENHO DE GOTEJADORES EM FUNÇÃO DA APLICAÇÃO DE TURFA GEL NA ÁGUA DE IRRIGAÇÃO
}

Lucas de Oliveira Gomes ${ }^{1}$, Anthony Wellington Almeida Gomes ${ }^{1}$, Renato Campos de Oliveira $^{2}$, Marconi Batista Teixeira ${ }^{2 *}$, Allan Cunha Barros ${ }^{1}$, Rubens Duarte Coelho ${ }^{1}$

\section{RESUMO}

O experimento foi conduzido no Laboratório de Irrigação do Departamento de Engenharia de Biossistemas - ESALQ/USP. Objetivou-se com este trabalho analisar o desempenho hidráulico (variação de vazão) de 26 modelos de gotejadores frente à aplicação de turfa gel via água de irrigação. O ensaio foi conduzido em três etapas: seis aplicações com a dosagem mínima recomendada pelo fabricante; duas aplicações com o dobro da dosagem recomendada pelo fabricante; e uma aplicação de cloro livre ( $\mathrm{pH}$ 5,5-6,0) e passagem de "pigs" (pedaço de esponja) com final de linha aberto. O delineamento experimental adotado foi o de blocos casualizados, sendo utilizados os testes "F" para análise de variância e Tukey a 5\% de significância para comparação de médias. Foi utilizado o método gravimétrico para coleta da vazão dos gotejadores na linha para cada modelo por um período de cinco minutos. Posteriormente, foram calculados vazão média, coeficiente de variação de vazão $(\mathrm{CV})$ e uniformidade de distribuição de água (UD). A utilização de uma dosagem equivalente ao dobro do mínimo recomendado pelo fabricante permitiu acelerar o processo de obstrução de quatro modelos testados (modelos 11, 12, 15 e 16). No entanto, o produto pode ser utilizado via água de irrigação por gotejamento na concentração mínima recomendada pelo fabricante.

Palavras-Chave: dosagem, tempo de exposição, fertirrigação, coeficiente de variação.

\section{DRIPPERS PERFORMANCE IN FUCTION APPLICATION OF PEAT GEL IN IRRIGATION WATER}

\footnotetext{
ABSTRACT

The experiment was carried at the Irrigation Laboratory of University of São Paulo. This work sought to quantify possible flow disturbs in 26 drip models submitted to the treatment with water containing peat gel. The trial was conducted in three stages: six

${ }^{1}$ Departamento de Engenharia de Biossistemas - LEB - ESALQ/USP, Av. Pádua Dias, 11, Agronomia, Caixa Postal 9, Piracicaba - SP. CEP: 13.418-900.

${ }^{2}$ Instituto Federal Goiano - Câmpus Rio Verde, Rod. Sul Goiana, Km 01, Zona Rural, Caixa Postal 66, Rio Verde-GO. CEP: 75.901-970. *Autor para correspondência. E-mail: marconibt@gmail.com
} 


\section{DESEMPENHO DE GOTEJADORES EM FUNÇÃO DA APLICAÇÃO DE TURFA GEL NA ÁGUA DE IRRIGAÇÃO}

applications with the minimum dosage recommended by the manufacturer, two applications at twice the dosage recommended by the manufacturer, and application of free chlorine ( $\mathrm{pH} 5,5-$ $6,0)$ and passage of "pigs" (piece of sponge) with open end of line. Randomized blocks was the experimental delimitation adopted and was used the " $F$ " tests to the variance analysis and Tukey test with 5\% probability to averages comparisons and regression analysis. The gravimetric method was adopted to measure the drippers flow by five minutes of flow collect each line model. The obtained values were used to calculate: average flow (qm), flow rate coefficient variation (CV) and water uniformity distribution (UD). The use of the double of the minimum dosage recommended by the manufacturer allowed accelerating the obstruction process in the 11, 12, 15 and 16 models. However, the product can be used through drip irrigation water in the minimum concentration recommended by the manufacturer.

Keywords: dosage, exposure time, fertirrigation, coefficient variation.

\section{INTRODUÇÃO}

Nas últimas décadas, o mundo vem aumentando sua demanda por alimentos, provocando uma grande pressão econômica, fazendo com que os produtores busquem formas de produção cada vez mais eficientes quanto ao uso de insumos e respeito ao meio ambiente.

O uso de produtos orgânicos como insumos agrícolas promovem uma série de ações físicas, químicas e biológicas benéficas para as plantas e o solo. Entre estes produtos, destaca-se a turfa gel, que tem em sua composição substâncias húmicas, que são frações da matéria orgânica que podem ser consideradas resultado das últimas transformações da matéria orgânica. A turfa gel é formada por alguns ácidos como o ácido húmico, ácido fúlvico e o ácido hymatomelânico, este de menor importância (HANDRECK \& BLACK, 1999; MELO et al., 2008).

A estrutura química das substâncias húmicas é bastante desconhecida apesar dos constantes esforços em pesquisas nesta direção. A diferença mais importante entre os ácidos húmicos e fúlvicos é que os primeiros são colóides orgânicos muito complexos e os demais compostos de peso molecular relativamente baixo. Isto implica grande diferença de solubilidade (HANDRECK \& BLACK, 1999).

Excetuando o tamanho da molécula, trata-se de compostos orgânicos bastante similares, com um conteúdo de carbono entre $40 \%$ e $60 \%$, maior nos ácidos húmicos, e de oxigênio entre 30 e $50 \%$, maior nos ácidos fúlvicos. Este maior conteúdo de oxigênio dos ácidos fúlvicos implica uma maior riqueza em grupos oxigenados relacionados com os processos de quelatação de metais (HANDRECK \& BLACK, 1999; MELO et al., 2008).

Um dos sistemas de irrigação mais apropriados e em notável expansão é o sistema de irrigação por gotejamento, que apresenta vantagens como economia de água e energia, possibilidade de automação e de fertirrigação (SOUSA et al., 2011).

A turfa gel, pela sua grande quantidade de nutrientes, é altamente indicada para o uso em fertirrigação, desde que o sistema de irrigação seja por gotejamento (MELO et al., 2008).

Uma característica inerente ao sistema de irrigação por gotejamento é a pequena área de passagem da água nos emissores (pequenos diâmetros de orifício), o que pode ocasionar problemas de entupimento (GILBERT; FORD, 1986; KELLER; BLIESNER, 1990; PITTS et al., 1996) por uma combinação de fatores físicos, químicos e biológicos que caracterizam a qualidade da água utilizada na irrigação que, somados à prática de fertirrigação, podem agravar o problema. A obstrução de emissores afeta a uniformidade de aplicação de água e, 


\section{DESEMPENHO DE GOTEJADORES EM FUNÇÃO DA APLICAÇÃO DE TURFA GEL NA ÁGUA DE IRRIGAÇÃO}

consequentemente, reduz a eficiência da aplicação de produtos químicos.

Sagi et al. (1995) encontraram redução de vazão média de gotejadores igual a $38 \%$ em relação à vazão inicial em função da presença de mucilagem formada por colônias do protozoário Epystilus balanarum, que reduziu em $57 \%$ a área de passagem do emissor.

Estudando o efeito de diâmetros de orifício na taxa de entupimento de microaspersores, Boman et al. (1995) observaram que $20 \%$ dos emissores de $0,76 \mathrm{~mm}$ de diâmetro precisaram de limpeza, comparados a $14 \%$ dos emissores com 1,02 mm, a 7\% dos emissores com $1,27 \mathrm{~mm}$ e a $5 \%$ dos emissores com diâmetro de 1,52 mm. Para esse estudo, $46 \%$ dos casos de entupimento foram devidos a algas, 34\% obstrução por formigas e aranhas, $16 \%$ obstrução por lesmas e 4\% obstrução física por partículas de areia e pedaços de PVC.

$\mathrm{O}$ entupimento parcial dos emissores apresenta o inconveniente adicional de não ser perceptível visualmente, o que retarda a

\section{MATERIAL E MÉTODOS}

$\mathrm{O}$ experimento foi conduzido em uma bancada de ensaios com $11,0 \mathrm{~m}$ de comprimento por $4,0 \mathrm{~m}$ de largura e 5,80 $\mathrm{m}$ de altura, instalada no Laboratório de Irrigação do Departamento de Engenharia de Biossistemas, da Escola Superior de Agricultura "Luiz de Queiroz" (ESALQ/USP), situada no município de Piracicaba-SP. tomada de decisão para solução do problema (RESENDE et al., 2000). Segundo Teixeira et al. (2008), para prevenir o entupimento dos emissores, a avaliação sistemática do sistema de irrigação e o monitoramento da qualidade da água são essenciais.

Diferentes tipos de gotejadores apresentam graus de suscetibilidade diversos ao entupimento, seja em função de características construtivas (mecanismo de autolimpeza) e/ou hidráulicas. Neste sentido, a escolha do tipo de emissor e sistema de filtragem, somados ao manejo da irrigação adequado, tornam-se fatores decisivos no elenco de medidas a serem consideradas quando a água utilizada apresente parâmetros de qualidade que possam ocasionar problemas de entupimento.

Objetivou-se com este trabalho analisar o desempenho hidráulico (variação de vazão) de 26 modelos de gotejadores autocompensantes existentes no comércio, submetidos à aplicação de turfa gel via água de irrigação.

O sistema de aplicação permitia a recirculação da solução aplicada conforme o seguinte esquema: reservatório $\rightarrow$ gotejadores $\rightarrow$ telha $\rightarrow$ calha $\rightarrow$ reservatório.

$\mathrm{Na}$ Tabela 1 é apresentada a listagem dos emissores utilizados no ensaio, com o nome de seus respectivos fabricantes, suas características técnicas e a vazão mais comercializada no mercado nacional. 


\section{DESEMPENHO DE GOTEJADORES EM FUNÇÃO DA APLICAÇÃO DE TURFA} GEL NA ÁGUA DE IRRIGAÇÃO

Tabela 1. Características técnicas dos modelos, fluxo, vazão, diâmetro nominal ( $\varnothing \mathrm{N})$ e distância entre gotejadores (DEG)

\begin{tabular}{cllcccc}
\hline Identificação & Fabricante & \multicolumn{1}{c}{ Modelo } & Fluxo & Vazão $\left(\mathrm{L} \mathrm{h}^{-1}\right)$ & $\varnothing \mathrm{N}(\mathrm{mm})$ & DEG $(\mathrm{m})$ \\
\hline 1 & Naandan & Naantif PC & AC & 1,6 & 16 & 0,50 \\
2 & Naandan & Naan Paz & NC & 2,0 & 16 & 0,75 \\
3 & Naandan & Naan PC & AC & 2,1 & 16 & 0,80 \\
4 & Naandan & Naan PC & AC & 3,8 & 16 & 0,90 \\
5 & Plastro & Hydrogol & NC & 3,0 & 17 & 0,40 \\
6 & Netafim & Ram & AC & 2,3 & 16 & 0,50 \\
7 & Netafim & Ram & AC & 2,3 & 17 & 0,50 \\
8 & Plastro & HydroPc & AC & 2,0 & 17 & 0,80 \\
9 & Plastro & HydroPc & AC & 2,2 & 17 & 0,80 \\
10 & Plastro & HydroPc & AC & 2,2 & $16 / 25$ & 0,75 \\
11 & Plastro & HydroPcNd & AC & 2,35 & $16 / 40$ & 0,80 \\
12 & Plastro & HydroDrip & AC & 2,0 & 16 & 0,80 \\
13 & Amanco & CarboDrip & AC & 2,0 & 17 & 0,80 \\
14 & Netafim & Tiran & NC & 2,05 & 16 & 0,75 \\
15 & Irrimon & Twin Plus & AC & 1,8 & 17,5 & 1,00 \\
16 & Irrimon & VipLine & AC & 3,6 & 16 & 1,00 \\
17 & Irrimon & Irridrip Plus & AC & 2,5 & 16 & 1,00 \\
18 & Netafim & SuperTyphoon & NC & 2,0 & 16 & 0,60 \\
19 & Azud & Azudline & NC & 1,4 & 17 & 0,50 \\
20 & Toro Ag & Drip In & AC & 2,5 & 17 & 0,75 \\
21 & Petroísa & Petrodrip & NC & 1,5 & 16 & 0,30 \\
22 & Amanco & CarboDripCd/Ac & NC & 2,2 & 16 & 0,50 \\
23 & Irrimon & Irriloc & AC & 1,1 & 16,4 & 0,40 \\
24 & Queen Gil & Queen Gil & NC & 4,0 & 16,5 & 0,10 \\
25 & Netafim & Drip Net & AC & 1,6 & 16 & 0,75 \\
26 & Netafim & Uniran & AC & 1,6 & 16 & 0,33 \\
\hline
\end{tabular}

* AC - Autocompensante; NC - Não compensante.

O conjunto motobomba utilizado nos ensaios foi composto por uma bomba centrífuga, modelo KSB Megabloc 32160.1R. Para a retenção de partículas sólidas foi utilizado um filtro de disco de 100 mesh. As linhas gotejadoras foram unidas no início e ao final por registros de $1 / 2$ ' e conexões de PVC. Foi instalada uma tomada de pressão para monitoramento a cada medição de vazão. Para tanto, utilizou-se um manômetro de bourdon com faixa de leitura de $0-700 \mathrm{kPa}$.

Primeiramente foi realizada uma leitura de vazão $(0$ h) com água tratada provinda do sistema de abastecimento da ESALQ/USP, com o intuito de verificar a real condição hidráulica de cada modelo de gotejador ensaiado.
Posteriormente, procedeu-se à aplicação de turfa gel utilizando-se a concentração mínima recomendada pelo fabricante para diluição do produto em água: um litro de turfa gel para cada 300 litros de água (leituras de vazão: $48 \mathrm{~h}$ até 288 h). Nas leituras de vazão 336 e 384 h utilizou-se o dobro da concentração mínima: dois litros de turfa gel para cada 300 litros de água.

$\mathrm{O}$ procedimento de aplicação consistiu de 12 h de aplicação da solução + $36 \mathrm{~h}$ de descanso da solução na linha lateral. Após esse tempo era realizado a leitura de vazão de dez gotejadores em cada linha lateral dos modelos ensaiados.

A última avaliação consistiu de uma aplicação de 150 ppm de cloro livre (hipoclorito de sódio) + ácido fosfórico 


\section{DESEMPENHO DE GOTEJADORES EM FUNÇÃO DA APLICAÇÃO DE TURFA GEL NA ÁGUA DE IRRIGAÇÃO}

$85 \%$ P.A. para manutenção do $\mathrm{pH}$ entre 5,5 e $6,0+300 \mathrm{kPa}$, associado ao uso de "pigs" (pedaço de esponja) com final de linha aberto para retirada de possíveis incrustações nas linhas laterais, com o intuito de recuperação de vazão. Após tal procedimento, fez-se a leitura de vazão "Cloro + pigs".

$\mathrm{Na}$ Tabela 2 são apresentadas as características físico-químicas da turfa gel utilizada no ensaio.

Tabela 2. Características físico-químicas da turfa gel aplicada no sistema

\begin{tabular}{lcc}
\multicolumn{1}{c}{ Parâmetro } & Unidade & Quantidade \\
\hline $\begin{array}{l}\text { Carbono orgânico total }(\mathrm{C}) \text { - Solúvel em } \\
\text { água }\end{array}$ & $\mathrm{g} \mathrm{L}^{-1}$ & 67,2 \\
$\begin{array}{l}\text { Potássio total }\left(\mathrm{K}_{2} \mathrm{O}\right) \text { - Complexado por } \\
\text { substâncias húmicas }\end{array}$ & $\mathrm{g} \mathrm{L}^{-1}$ & 56,0 \\
$\begin{array}{l}\text { Enxofre total }(\mathrm{S}) \text { - Solúvel em água } \\
\text { Indice de salinidade }\end{array}$ & $\mathrm{g} \mathrm{L}^{-1}$ & 1,68 \\
$\begin{array}{l}\text { Densidade } \\
\text { pH }\end{array}$ & - & 1,0 \\
Condutividade elétrica & $\mathrm{dm} \mathrm{cm}^{-3}$ & 1,12 \\
Solubilidade em água a $20^{\circ} \mathrm{C}$ & - & 8,8 \\
& $\mathrm{mS} \mathrm{cm}^{-1}$ & 0,01 \\
& - & $100 \%$ \\
\hline
\end{tabular}

A operacionalização do funcionamento da bancada, quanto ao horário de início e de parada, foi feita manualmente, obedecendo rigorosamente aos horários de aplicação e leitura.

A água utilizada no ensaio proveio

do sistema de abastecimento da
ESALQ/USP. A Tabela 3 apresenta a análise química da água contendo o dobro da dosagem de turfa gel recomendada pelo fabricante, referente à leitura de vazão 384 h.

Tabela 3. Análise química da água do laboratório de irrigação - ESALQ/USP, contendo turfa gel diluída, referente à leitura de vazão $384 \mathrm{~h}$

\begin{tabular}{lcc}
\hline \multicolumn{1}{c}{ Parâmetro } & Unidade & Quantidade \\
\hline Alcalinidade $\left(2 \mathrm{CO}_{3}{ }^{2}+\mathrm{HCO}_{3-}\right)$ & $\mathrm{mg} \mathrm{L}^{-1}$ & 201,40 \\
Cloreto $\left(\mathrm{Cl}^{-}\right)$ & $\mathrm{mg} \mathrm{L}^{-1}$ & 147,80 \\
Nitrato $\left({\left.\mathrm{N}-\mathrm{NO}_{3}\right)}^{2}\right)$ & $\mathrm{mg} \mathrm{L}^{-1}$ & 162,30 \\
Sulfato $\left(\mathrm{SO}_{4}{ }^{-}\right)$ & $\mathrm{mg} \mathrm{L}^{-1}$ & 236,50 \\
Fósforo $(\mathrm{P})$ & $\mathrm{mg} \mathrm{L}^{-1}$ & 0,38 \\
Nitrogênio Amoniacal $\left(\mathrm{N}-N H_{3}\right)$ & $\mathrm{mg} \mathrm{L}^{-1}$ & 19,94 \\
Sódio $\left(\mathrm{Na}^{+}\right)$ & $\mathrm{mg} \mathrm{L}^{-1}$ & 3720,0 \\
Potássio $\left(\mathrm{K}^{+}\right)$ & $\mathrm{mg} \mathrm{L}^{-1}$ & 3560,0 \\
Cálcio $\left(\mathrm{Ca}^{2+}\right)$ & $\mathrm{mg} \mathrm{L}^{-1}$ & 22,60 \\
Magnésio $\left(\mathrm{Mg}^{2+}\right)$ & $\mathrm{mg} \mathrm{L}^{-1}$ & 1,70 \\
Ferro $(\mathrm{Fe})$ & $\mathrm{mg} \mathrm{L}$ & 0,40 \\
Cobre $(\mathrm{Cu})$ & $\mathrm{mg} \mathrm{L}^{-1}$ & 0,15 \\
Manganês $(\mathrm{Mn})$ & $\mathrm{mg} \mathrm{L}^{-1}$ & 0,03 \\
Zinco $(\mathrm{Zn})$ & $\mathrm{mg} \mathrm{L}$ & 0,19 \\
Cor Aparente & $\mathrm{PtCo}^{-1}$ & 504 \\
Turbidez & $\mathrm{FTU}^{-1}$ & 96 \\
Sedimentos em suspensão & $\mathrm{mg} \mathrm{L}^{-1}$ & 2046,70 \\
Condutividade Elétrica $(\mathrm{CE})$ & $\mathrm{mS} \mathrm{cm}^{-1}$ & 0,80 \\
pH & & 7,90 \\
Gás Carbônico $\left(\mathrm{CO}_{2}\right)$ & $\mathrm{mg} \mathrm{L}^{-1}$ & 4,20 \\
\hline
\end{tabular}




\section{DESEMPENHO DE GOTEJADORES EM FUNÇÃO DA APLICAÇÃO DE TURFA GEL NA ÁGUA DE IRRIGAÇÃO}

Acidez

$\mathrm{mg} \mathrm{L}^{-1}$

20,10

Dureza Total* $\left(\mathrm{CaCO}_{3}\right)$

$\mathrm{mg} \mathrm{L^{-1 }}$

64,70

* Dureza Total calculada com base no equivalente de carbonato de cálcio $\left(\mathrm{CaCO}_{3}\right)$ segundo Franson (1995): 2,497 [Ca, mg L $\left.{ }^{-1}\right]+4,118\left[\mathrm{Mg}, \mathrm{mg} \mathrm{L}^{-1}\right]$. Franson, M.A.H. Standard methods for the examination of water and wastewater.American Public Health Association, Washington, 19ª Edição, 1995.

O procedimento para leitura individual da vazão dos gotejadores consistiu da pressurização do sistema (150 $\mathrm{kPa})$, posicionamento de recipientes $(1$ litro) apoiados sobre placas de PVC de 25 x $15 \mathrm{~cm}^{2}$ sob os respectivos gotejadores com uma defasagem de 5 segundos, retirada sequencial dos recipientes após 5 minutos também com defasagem de 5 segundos, transporte dos recipientes para a bancada de pesagem, pesagem e tabulação dos dados.

Para maior exatidão na determinação do volume coletado de cada emissor, foi utilizado o método gravimétrico, expressando-se os valores de vazão em L $\mathrm{h}^{-1}$. Foi utilizada uma balança de precisão certificada (OHAUS) com precisão de 0,01 g.

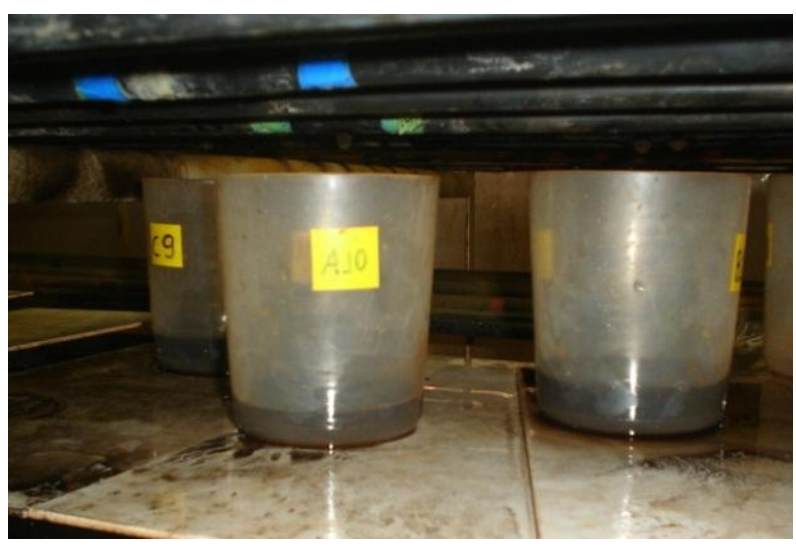

Figura 1a. Coleta de água.

Depois de tabulados os pesos, foram feitos os cálculos da vazão, do coeficiente de variação de vazão e da uniformidade de

$$
q=\frac{P}{1000 t} 60
$$

em que:

$$
\mathrm{q} \text { - vazão do gotejador, } \mathrm{L} \mathrm{h}^{-1} \text {; }
$$

Para realizar-se a leitura de vazão das linhas gotejadoras, utilizou-se a água provinda de um reservatório com água isenta de produtos químicos. A temperatura da água foi monitorada durante as leituras de vazão. Uma adaptação no tubo de $100 \mathrm{~mm}$ conectado ao fundo da calha permitiu descartar a água utilizada para limpeza das linhas gotejadoras em um canal dentro do Laboratório de Irrigação, utilizado somente para descarte de água proveniente de ensaios.

Nas Figuras 1a e 1b são mostrados os acessórios - placa de PVC, recipiente de plástico e balança digital, utilizados para a coleta do volume de água no tempo prédeterminado.

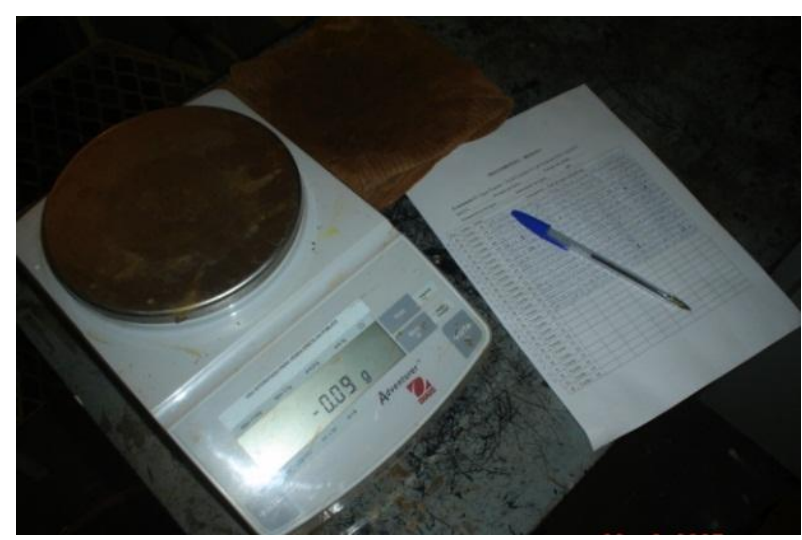

Figura 1b. Método gravimétrico.

distribuição de água pelas seguintes equações: 


\section{DESEMPENHO DE GOTEJADORES EM FUNÇÃO DA APLICAÇÃO DE TURFA} GEL NA ÁGUA DE IRRIGAÇÃO

$\mathrm{P}$ - peso da água coletada, $\mathrm{g}$; e

$\mathrm{t}$ - tempo de coleta, min.

$$
C V_{q}=\frac{s}{q} 100
$$

em que:

$\mathrm{CV}_{q}$ - coeficiente de variação de vazão, \%;

$s$ - desvio padrão da vazão dos gotejadores, $\mathrm{L} \mathrm{h}^{-1} ; \mathrm{e}$

$\bar{q}$ - vazão média dos gotejadores, $\mathrm{L} \mathrm{h}^{-1}$.

$$
U D=\frac{q_{25 \%}}{\bar{q}} 100
$$

em que:

UD - uniformidade de distribuição de água, \%;

$\mathrm{q}_{25 \%}$ - vazão média de $1 / 4$ dos menores valores, $\mathrm{L} \mathrm{h}^{-1} ; \mathrm{e}$

Cálculos estequiométricos foram feitos para a determinação da dosagem de cloro livre utilizada no final do ensaio, e para efeito de monitoramento, amostras de água ao final das linhas laterais foram coletadas para determinar o teor de cloro livre, utilizando-se kits de medição de cloro usados no tratamento de piscinas, com a necessária diluição para atingir a faixa de leitura do kit, a qual é de 1 a $5 \mathrm{mg}$ $\mathrm{L}^{-1}$.

Para o cálculo da dosagem de ácido fosfórico $85 \%$ P.A. utilizada para manter o $\mathrm{pH}$ na faixa de 5,5 a 6,0 , foi feita uma curva de titulação.

Para diminuir a perda de solução por respingo fora da bancada, foram colocadas cortinas laterais, com intuito também de proteger a bancada contra corrosão, e seus operadores utilizaram vestimentas especiais para manuseio do ácido e do cloro durante o ensaio.

$\mathrm{O}$ delineamento adotado foi blocos casualizados, com utilização do teste " $F$ " para análise de variância e Tukey, a $5 \%$ de significância, para comparação de médias.

\section{RESULTADOS E DISCUSSÃO}

Os 26 modelos de gotejadores ensaiados foram separados em quatro grupos de acordo com a faixa de vazão que operam (Tabela 4).

Tabela 4. Classificação dos modelos de gotejadores segundo a vazão nominal $\left(\mathrm{L} \mathrm{h}^{-1}\right)$

\begin{tabular}{ccc}
\hline Grupo & Vazão nominal $\left(\mathrm{L} \mathrm{h}^{-1}\right)$ & Modelos de gotejadores \\
\hline 1 & 1,0 a 1,6 & $1-19-21-23-24-25-26$ \\
2 & 1,8 a 2,05 & $2-8-12-13-14-15-18$ \\
3 & 2,1 a 2,35 & $3-6-7-9-10-11-22$ \\
4 & 2,5 a 4,0 & $4-5-16-17-20$ \\
\hline
\end{tabular}

Os modelos de emissores mostraram comportamentos variáveis com a aplicação de turfa gel, concordando com Ribeiro et al. (2010), que, avaliando gotejadores submetidos à aplicação de cloreto de potássio (branco e vermelho) via duas qualidades de água, sugeriram ser a arquitetura interna dos gotejadores um fator determinante na caracterização do processo de entupimento.

Nas Tabelas 5 e 6 são apresentados os valores de vazão média $\left(\mathrm{L} \mathrm{h}^{-1}\right)$, quantificados pela medição individual de vazão para os diferentes modelos utilizados 


\section{DESEMPENHO DE GOTEJADORES EM FUNÇÃO DA APLICAÇÃO DE TURFA} GEL NA ÁGUA DE IRRIGAÇÃO

no ensaio e testes de média, respectivamente.

Tabela 5. Vazão média expressa em $\mathrm{L} \mathrm{h}^{-1}$ dos gotejadores avaliados, no período do ensaio, para os emissores dos Grupos 1 e 2, e testes de média, respectivamente

\begin{tabular}{ccccccccc}
\hline \multirow{2}{*}{$\begin{array}{c}\text { Aplicações } \\
\text { (horas) }\end{array}$} & \multirow{2}{*}{ Avaliações } & \multicolumn{7}{c}{ Emissores do Grupo 1} \\
\cline { 3 - 8 } & 0 & $0,50^{\mathrm{a}}$ & $1,19^{\mathrm{a}}$ & $1,27^{\mathrm{ab}}$ & $0,94^{\mathrm{a}}$ & $0,52^{\mathrm{a}}$ & $1,51^{\mathrm{a}}$ & $1,45^{\mathrm{ab}}$ \\
48 & 1 & $0,58^{\mathrm{a}}$ & $1,38^{\mathrm{a}}$ & $1,24^{\mathrm{ab}}$ & $0,96^{\mathrm{a}}$ & $0,61^{\mathrm{a}}$ & $1,63^{\mathrm{a}}$ & $1,58^{\mathrm{a}}$ \\
96 & 2 & $0,63^{\mathrm{a}}$ & $1,27^{\mathrm{a}}$ & $1,42^{\mathrm{a}}$ & $0,81^{\mathrm{a}}$ & $0,42^{\mathrm{a}}$ & $1,61^{\mathrm{a}}$ & $1,45^{\mathrm{ab}}$ \\
144 & 3 & $0,49^{\mathrm{a}}$ & $1,20^{\mathrm{a}}$ & $0,53^{\mathrm{bc}}$ & $0,35^{\mathrm{a}}$ & $0,59^{\mathrm{a}}$ & $1,64^{\mathrm{a}}$ & $1,48^{\mathrm{ab}}$ \\
192 & 4 & $0,42^{\mathrm{a}}$ & $1,42^{\mathrm{a}}$ & $0,38^{\mathrm{c}}$ & $0,30^{\mathrm{a}}$ & $0,55^{\mathrm{a}}$ & $1,62^{\mathrm{a}}$ & $1,01^{\mathrm{abc}}$ \\
240 & 5 & $0,47^{\mathrm{a}}$ & $1,01^{\mathrm{a}}$ & $0,29^{\mathrm{c}}$ & $0,40^{\mathrm{a}}$ & $0,55^{\mathrm{a}}$ & $1,60^{\mathrm{a}}$ & $1,11^{\mathrm{abc}}$ \\
288 & 6 & $0,45^{\mathrm{a}}$ & $1,12^{\mathrm{a}}$ & $0,28^{\mathrm{c}}$ & $0,45^{\mathrm{a}}$ & $0,42^{\mathrm{a}}$ & $1,66^{\mathrm{a}}$ & $0,97^{\mathrm{abc}}$ \\
336 & 7 & $0,29^{\mathrm{a}}$ & $0,84^{\mathrm{a}}$ & $0,51^{\mathrm{bc}}$ & $0,27^{\mathrm{a}}$ & $0,31^{\mathrm{a}}$ & $1,57^{\mathrm{a}}$ & $0,90^{\mathrm{abc}}$ \\
384 & 8 & $0,27^{\mathrm{a}}$ & $0,90^{\mathrm{a}}$ & $0,23^{\mathrm{c}}$ & $0,28^{\mathrm{a}}$ & $0,56^{\mathrm{a}}$ & $1,62^{\mathrm{a}}$ & $0,45^{\mathrm{c}}$ \\
Cloro + pigs & 9 & $0,25^{\mathrm{a}}$ & $1,21^{\mathrm{a}}$ & $0,45^{\mathrm{bc}}$ & $0,48^{\mathrm{a}}$ & $0,47^{\mathrm{a}}$ & $1,63^{\mathrm{a}}$ & $0,84^{\mathrm{bc}}$ \\
\hline
\end{tabular}

\begin{tabular}{ccccccccc}
\hline \multirow{2}{*}{$\begin{array}{c}\text { Aplicações } \\
\text { (horas) }\end{array}$} & \multirow{2}{*}{ Avaliações } & \multicolumn{7}{c}{ Emissores do Grupo 2} \\
\cline { 3 - 8 } & & 2 & 8 & 12 & 13 & 14 & 15 & 18 \\
\hline 0 & 0 & $2,26^{\mathrm{a}}$ & $1,87^{\mathrm{a}}$ & $2,05^{\mathrm{a}}$ & $1,83^{\mathrm{a}}$ & $1,92^{\mathrm{a}}$ & $1,77^{\mathrm{a}}$ & $1,92^{\mathrm{a}}$ \\
48 & 1 & $2,21^{\mathrm{ab}}$ & $1,79^{\mathrm{a}}$ & $2,02^{\mathrm{a}}$ & $1,68^{\mathrm{a}}$ & $2,03^{\mathrm{a}}$ & $2,02^{\mathrm{a}}$ & $2,15^{\mathrm{a}}$ \\
96 & 2 & $1,79^{\mathrm{bcd}}$ & $1,67^{\mathrm{a}}$ & $1,95^{\mathrm{ab}}$ & $1,95^{\mathrm{a}}$ & $2,01^{\mathrm{a}}$ & $1,46^{\mathrm{ab}}$ & $1,99^{\mathrm{a}}$ \\
144 & 3 & $1,80^{\mathrm{bcd}}$ & $1,81^{\mathrm{a}}$ & $1,59^{\mathrm{ab}}$ & $2,01^{\mathrm{a}}$ & $2,10^{\mathrm{a}}$ & $1,43^{\mathrm{ab}}$ & $1,76^{\mathrm{a}}$ \\
192 & 4 & $1,44^{\mathrm{d}}$ & $1,55^{\mathrm{a}}$ & $1,34^{\mathrm{ab}}$ & $1,54^{\mathrm{a}}$ & $1,61^{\mathrm{a}}$ & $0,77^{\mathrm{cd}}$ & $1,24^{\mathrm{a}}$ \\
240 & 5 & $1,74^{\mathrm{cd}}$ & $1,67^{\mathrm{a}}$ & $1,63^{\mathrm{ab}}$ & $1,84^{\mathrm{a}}$ & $1,63^{\mathrm{a}}$ & $0,94^{\mathrm{bc}}$ & $1,74^{\mathrm{a}}$ \\
288 & 6 & $1,76^{\mathrm{cd}}$ & $1,64^{\mathrm{a}}$ & $1,37^{\mathrm{ab}}$ & $2,04^{\mathrm{a}}$ & $1,73^{\mathrm{a}}$ & $0,39^{\mathrm{cde}}$ & $1,55^{\mathrm{a}}$ \\
336 & 7 & $1,70^{\mathrm{cd}}$ & $1,49^{\mathrm{a}}$ & $0,88^{\mathrm{b}}$ & $1,81^{\mathrm{a}}$ & $1,64^{\mathrm{a}}$ & $0,02^{\mathrm{e}}$ & $1,40^{\mathrm{a}}$ \\
384 & 8 & $1,96^{\mathrm{abc}}$ & $1,52^{\mathrm{a}}$ & $0,90^{\mathrm{b}}$ & $1,60^{\mathrm{a}}$ & $1,52^{\mathrm{a}}$ & $0,12^{\mathrm{e}}$ & $1,62^{\mathrm{a}}$ \\
Cloro + pigs & 9 & $2,02^{\mathrm{abc}}$ & $1,84^{\mathrm{a}}$ & $1,48^{\mathrm{ab}}$ & $1,82^{\mathrm{a}}$ & $1,93^{\mathrm{a}}$ & $0,27^{\mathrm{de}}$ & $2,01^{\mathrm{a}}$ \\
\hline
\end{tabular}

* Média seguida de mesma letra na coluna não diferem significativamente pelo teste Tukey a $5 \%$ de probabilidade.

No grupo 1, observou-se que os modelos 21 e 26 foram os que apresentaram a menor redução de vazão quando a dosagem de turfa gel foi duplicada a partir de $336 \mathrm{~h}$ de ensaio até 384 h. Este comportamento não foi significativo para os demais modelos do grupo 1.
No grupo 2, o modelo 15 foi o que apresentou as menores vazões com obstrução severa quando a dosagem de turfa gel foi elevada em 100\% (leituras de vazão entre 336 e 384 h). 


\section{DESEMPENHO DE GOTEJADORES EM FUNÇÃO DA APLICAÇÃO DE TURFA} GEL NA ÁGUA DE IRRIGAÇÃO

Tabela 6. Vazão média expressa em $\mathrm{L} \mathrm{h}^{-1}$ dos gotejadores avaliados, no período do ensaio, para os emissores dos Grupos 3 e 4 e testes de média, respectivamente

\begin{tabular}{|c|c|c|c|c|c|c|c|c|}
\hline \multirow{2}{*}{$\begin{array}{c}\text { Aplicações } \\
\text { (horas) }\end{array}$} & \multirow{2}{*}{ Avaliações } & \multicolumn{7}{|c|}{ Emissores do Grupo 3} \\
\hline & & 3 & 6 & 7 & 9 & 10 & 11 & 22 \\
\hline 0 & 0 & $1,95^{\mathrm{a}}$ & $2,19^{\mathrm{a}}$ & $2,05^{\mathrm{a}}$ & $1,45^{\mathrm{a}}$ & $1,84^{\mathrm{a}}$ & $1,74^{\mathrm{a}}$ & $1,87^{\mathrm{abc}}$ \\
\hline 48 & 1 & $1,91^{\mathrm{a}}$ & $2,28^{\mathrm{a}}$ & $2,11^{\mathrm{a}}$ & $1,70^{\mathrm{a}}$ & $2,16^{\mathrm{a}}$ & $2,05^{\mathrm{a}}$ & $1,52^{\mathrm{c}}$ \\
\hline 96 & 2 & $1,81^{\mathrm{a}}$ & $2,30^{\mathrm{a}}$ & $2,01^{\mathrm{a}}$ & $1,64^{\mathrm{a}}$ & $2,02^{\mathrm{a}}$ & $1,61^{\mathrm{a}}$ & $2,03^{\mathrm{ab}}$ \\
\hline 144 & 3 & $1,45^{\mathrm{a}}$ & $2,33^{\mathrm{a}}$ & $1,88^{\mathrm{a}}$ & $1,66^{\mathrm{a}}$ & $1,94^{\mathrm{a}}$ & $1,84^{\mathrm{a}}$ & $1,80^{\mathrm{abc}}$ \\
\hline 192 & 4 & $1,86^{\mathrm{a}}$ & $2,02^{\mathrm{a}}$ & $1,70^{\mathrm{a}}$ & $1,57^{\mathrm{a}}$ & $1,95^{\mathrm{a}}$ & $1,65^{\mathrm{a}}$ & $1,83^{\mathrm{abc}}$ \\
\hline 240 & 5 & $2,07^{\mathrm{a}}$ & $2,18^{a}$ & $1,50^{\mathrm{a}}$ & $1,62^{\mathrm{a}}$ & $1,99^{\mathrm{a}}$ & $1,81^{\mathrm{a}}$ & $2,13^{\mathrm{a}}$ \\
\hline 288 & 6 & $1,68^{\mathrm{a}}$ & $2,05^{\mathrm{a}}$ & $1,84^{\mathrm{a}}$ & $1,41^{\mathrm{a}}$ & $2,12^{\mathrm{a}}$ & $1,89^{\mathrm{a}}$ & $1,99^{\mathrm{ab}}$ \\
\hline 336 & 7 & $1,41^{\mathrm{a}}$ & $1,74^{\mathrm{a}}$ & $1,63^{\mathrm{a}}$ & $1,37^{\mathrm{a}}$ & $1,84^{\mathrm{a}}$ & $1,55^{\mathrm{a}}$ & $1,75^{\mathrm{bc}}$ \\
\hline 384 & 8 & $1,59^{\mathrm{a}}$ & $1,74^{\mathrm{a}}$ & $1,31^{\mathrm{a}}$ & $0,99^{a}$ & $2,01^{\mathrm{a}}$ & $1,48^{\mathrm{a}}$ & $2,02^{\mathrm{ab}}$ \\
\hline Cloro + pigs & 9 & $1,69^{\mathrm{a}}$ & $2,04^{\mathrm{a}}$ & $1,67^{\mathrm{a}}$ & $1,36^{\mathrm{a}}$ & $1,95^{\mathrm{a}}$ & $1,83^{\mathrm{a}}$ & $2,14^{\mathrm{a}}$ \\
\hline \multirow{2}{*}{$\begin{array}{c}\text { Aplicações } \\
\text { (horas) }\end{array}$} & \multirow{2}{*}{ Avaliações } & \multicolumn{7}{|c|}{ Emissores do Grupo 4} \\
\hline & & 4 & 5 & 16 & 17 & 20 & & \\
\hline 0 & 0 & $3,82^{\mathrm{a}}$ & $2,57^{\mathrm{a}}$ & $2,11^{\mathrm{a}}$ & $2,35^{\mathrm{ab}}$ & $2,09^{\mathrm{ab}}$ & & \\
\hline 48 & 1 & $3,89^{\mathrm{a}}$ & $2,62^{\mathrm{a}}$ & $2,28^{a}$ & $2,64^{a}$ & $2,20^{\mathrm{ab}}$ & & \\
\hline 96 & 2 & $3,66^{\mathrm{a}}$ & $2,38^{\mathrm{ab}}$ & $0,89^{\mathrm{b}}$ & $2,35^{\mathrm{ab}}$ & $2,11^{\mathrm{ab}}$ & & \\
\hline 144 & 3 & $3,73^{\mathrm{a}}$ & $2,38^{\mathrm{ab}}$ & $0,66^{\mathrm{b}}$ & $2,25^{\mathrm{ab}}$ & $2,43^{\mathrm{a}}$ & & \\
\hline 192 & 4 & $3,47^{\mathrm{a}}$ & $2,26^{\mathrm{ab}}$ & $0,40^{\mathrm{b}}$ & $2,19^{\mathrm{ab}}$ & $2,03^{\mathrm{ab}}$ & & \\
\hline 240 & 5 & $3,48^{\mathrm{a}}$ & $2,32^{\mathrm{ab}}$ & $0,37^{\mathrm{b}}$ & $2,29^{\mathrm{ab}}$ & $2,05^{\mathrm{ab}}$ & & \\
\hline 288 & 6 & $3,89^{\mathrm{a}}$ & $1,99^{\mathrm{b}}$ & $0,08^{\mathrm{b}}$ & $1,74^{\mathrm{b}}$ & $1,89^{\mathrm{ab}}$ & & \\
\hline 336 & 7 & $3,02^{\mathrm{a}}$ & $1,95^{\mathrm{b}}$ & $0,08^{\mathrm{b}}$ & $1,87^{\mathrm{ab}}$ & $1,63^{\mathrm{b}}$ & & \\
\hline 384 & 8 & $3,01^{\mathrm{a}}$ & $2,36^{\mathrm{ab}}$ & $0,28^{\mathrm{b}}$ & $2,18^{\mathrm{ab}}$ & $0,93^{\mathrm{c}}$ & & \\
\hline Cloro + pigs & 9 & $3,16^{\mathrm{a}}$ & $2,64^{\mathrm{a}}$ & $0,50^{\mathrm{b}}$ & $2,43^{\mathrm{ab}}$ & $1,88^{\mathrm{ab}}$ & & \\
\hline
\end{tabular}

* Média seguida de mesma letra na coluna não diferem significativamente pelo teste Tukey a $5 \%$ de probabilidade.

No grupo 3, apenas o modelo 22 apresentou variação de vazão, tendo ocorrido um acréscimo de vazão acima do observado no início do ensaio. Os demais modelos deste grupo não tiveram alterações significativas na vazão.

Para o grupo 4, o modelo 16 foi o mais suscetível à obstrução devido ao uso de turfa gel via água de irrigação. $\mathrm{O}$ modelo 20 destacou-se com uma queda de vazão significativa após o uso de $100 \%$ da dosagem de turfa gel. No entanto, ocorreu recuperação de vazão após a aplicação do tratamento com cloro livre e com passagem de "pigs".

Nas Figuras 2, 3, 4 e 5 são mostrados os valores de coeficiente de variação de vazão para os modelos de gotejadores avaliados nos grupos de 1 a 4 . 
DESEMPENHO DE GOTEJADORES EM FUNÇÃO DA APLICAÇÃO DE TURFA GEL NA ÁGUA DE IRRIGAÇÃO

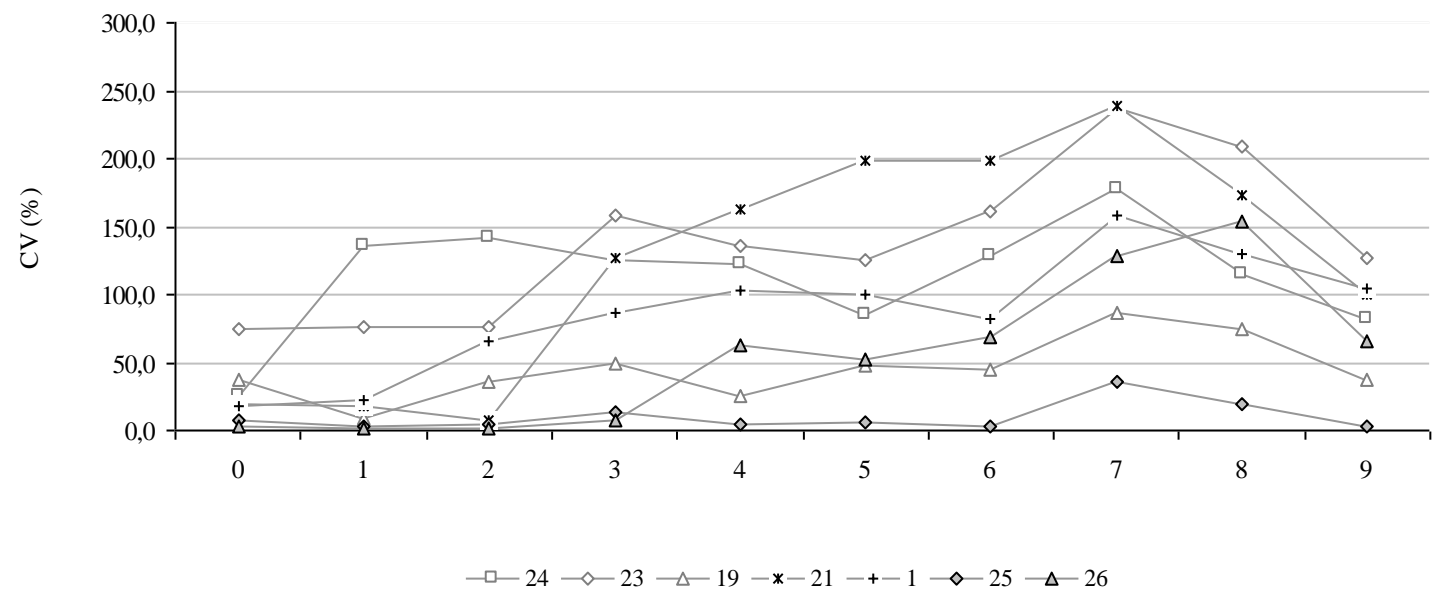

Figura 2. Coeficiente de variação de vazão para os modelos de gotejadores do grupo 1 .

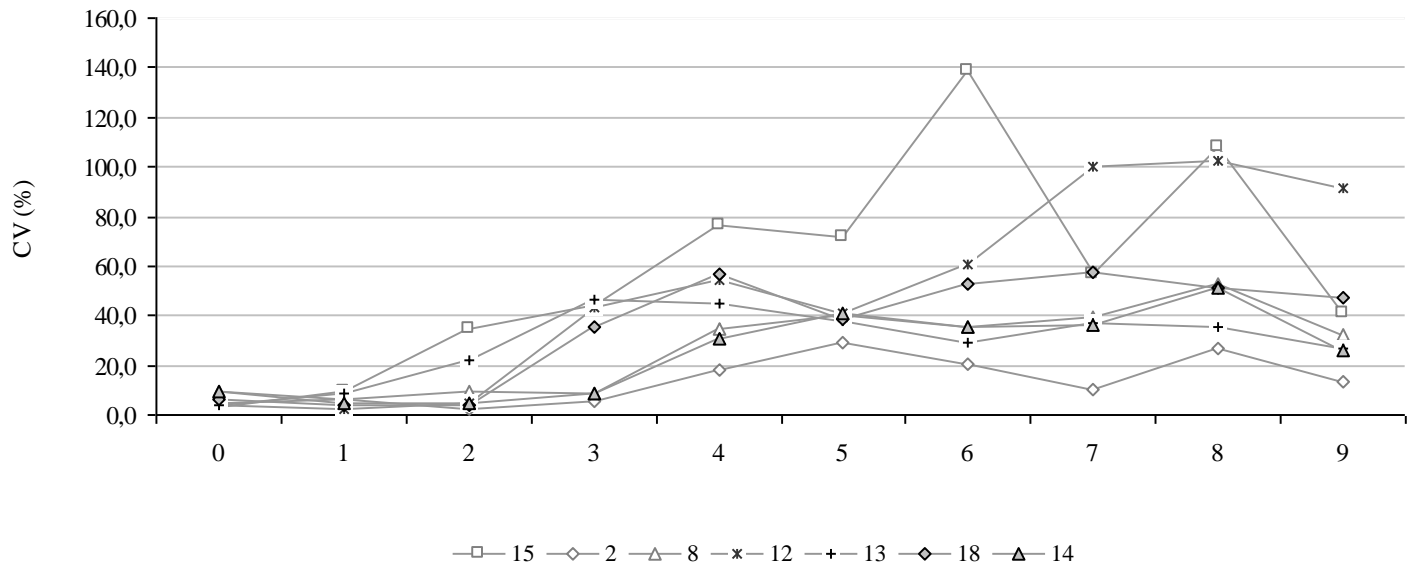

Figura 3. Coeficiente de variação de vazão para os modelos de gotejadores do grupo 2.

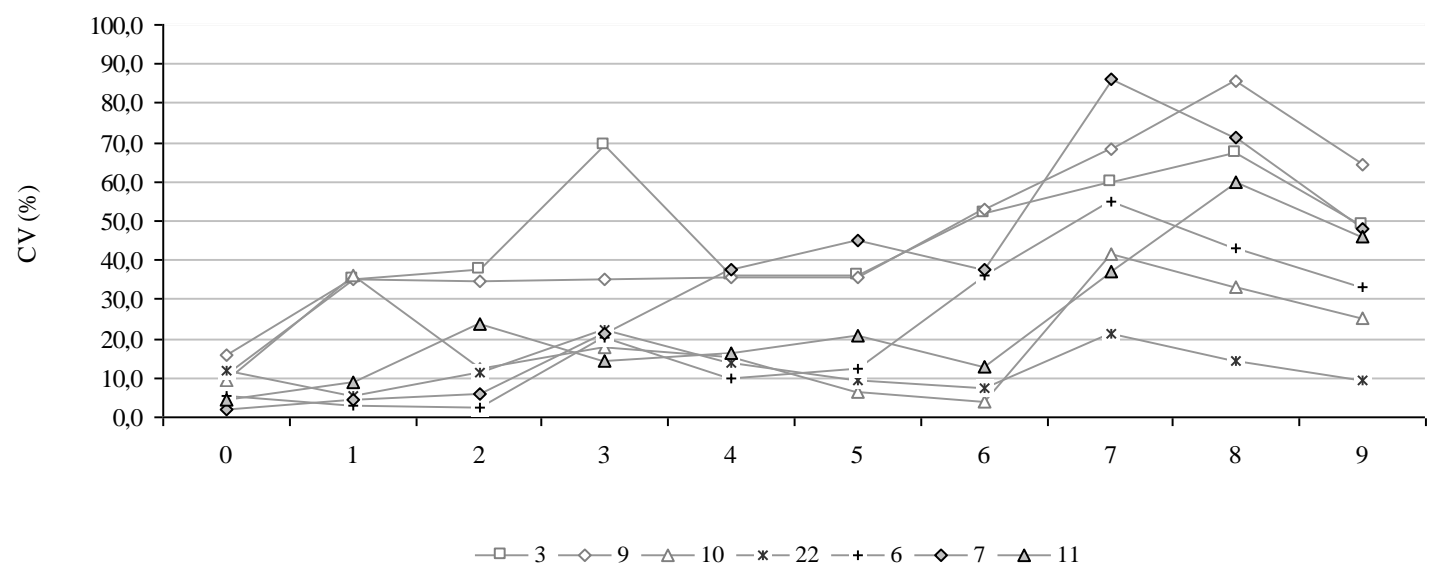

Rev. Bras. Agric. Irrigada v. 7, n⿳0. 1, p. 27 - 41 


\section{DESEMPENHO DE GOTEJADORES EM FUNÇÃO DA APLICAÇÃO DE TURFA GEL NA ÁGUA DE IRRIGAÇÃO}

Figura 4. Coeficiente de variação de vazão para os modelos de gotejadores do grupo 3.

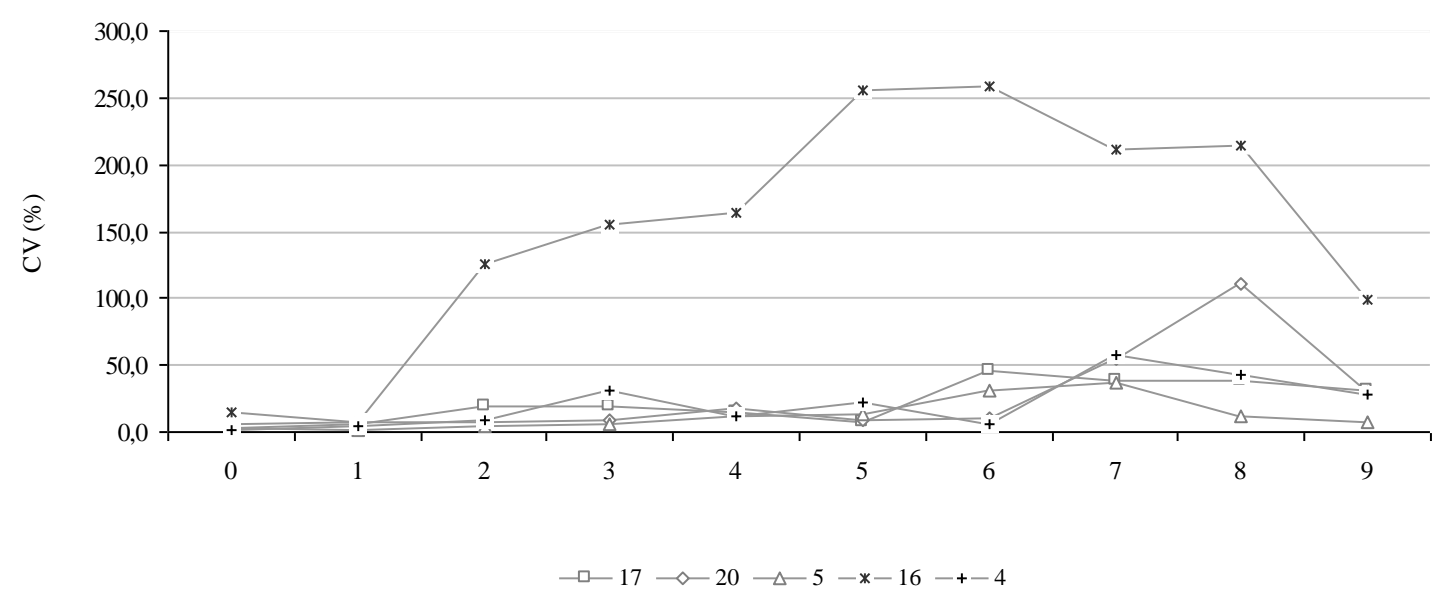

Figura 5. Coeficiente de variação de vazão para os modelos de gotejadores do grupo 4 .

Para o grupo 1 , o único modelo de gotejador que apresentou coeficiente de variação de vazão satisfatório foi o modelo 25. Os demais modelos apresentaram classificação inaceitável de acordo com Abreu et al. (1987).

No grupo 2, apenas o modelo 2 apresentou coeficiente de variação menor que $10 \%$, nas três primeiras aplicações de turfa gel. Isto mostra que todo o grupo 2 foi suscetível à aplicação de turfa gel via água de irrigação. $\mathrm{O}$ modelo 15 mostrou a maior variação de vazão durante o ensaio, porém notou-se recuperação após a aplicação de cloro livre e passagem de "pigs" nas linhas laterais.

Já os modelos de gotejadores avaliados tanto no grupo 3 quanto no grupo 4 apresentaram classificação inaceitável (Abreu et al., 1987), e o modelo 16 (grupo 4) foi o que apresentou os maiores valores de coeficiente de variação de vazão durante o ensaio, ultrapassando a faixa de $250 \%$.

Barros et al. (2009), avaliando o entupimento de gotejadores em função da aplicação de superfosfato simples e ácido nítrico, observaram após 360 horas coeficientes de variação de vazão variando entre 2 e $110 \%$, porém, a maioria dos modelos manteve níveis aceitáveis de variação, o que indica uma maior susceptibilidade de entupimento dos emissores disponíveis no mercado à aplicação com turfa gel, principalmente quando são utilizadas dosagens acima do recomendado.

Nas Figuras 6, 7, 8 e 9 são mostrados os valores de uniformidade de distribuição de água para os modelos de gotejadores avaliados nos grupos de 1 a 4 . 
DESEMPENHO DE GOTEJADORES EM FUNÇÃO DA APLICAÇÃO DE TURFA GEL NA ÁGUA DE IRRIGAÇÃO

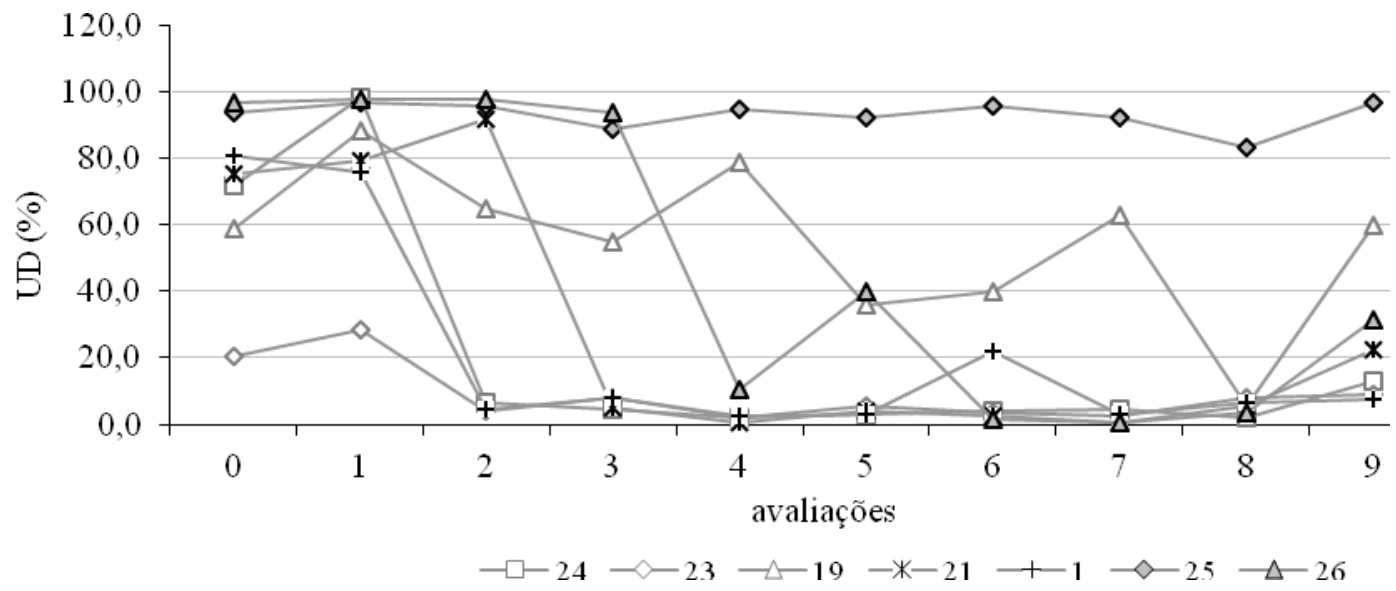

Figura 6. Uniformidade de distribuição de água para os modelos de gotejadores do grupo 1 .

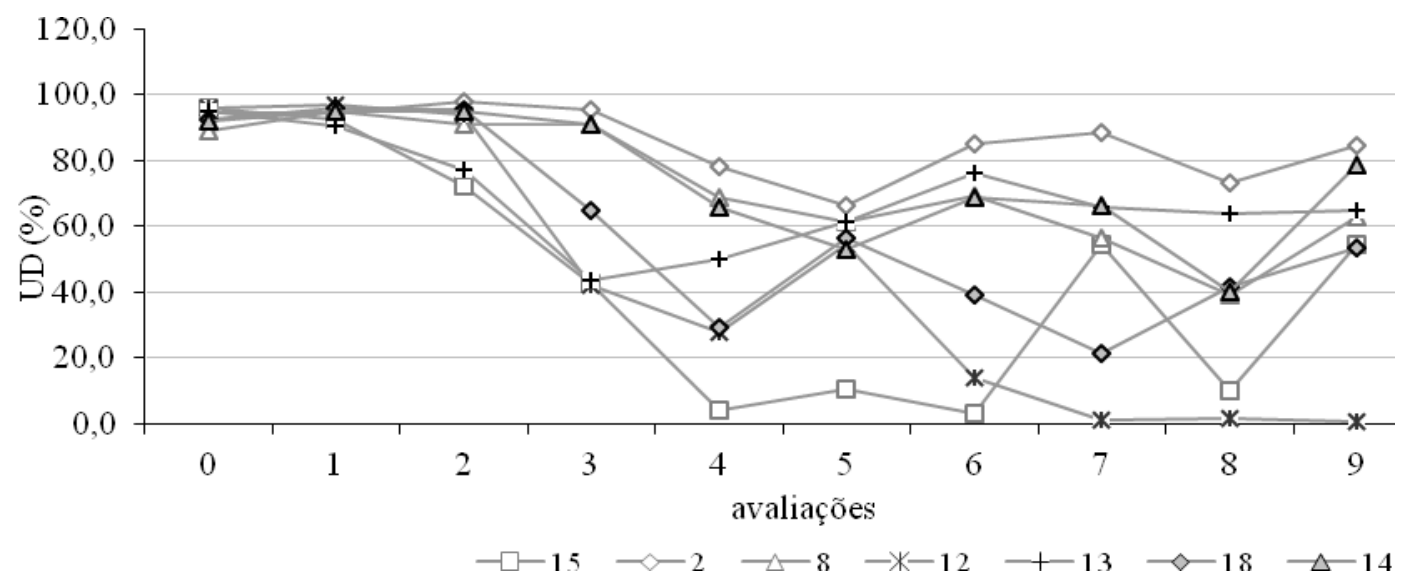

Figura 7. Uniformidade de distribuição de água para os modelos de gotejadores do grupo 2.

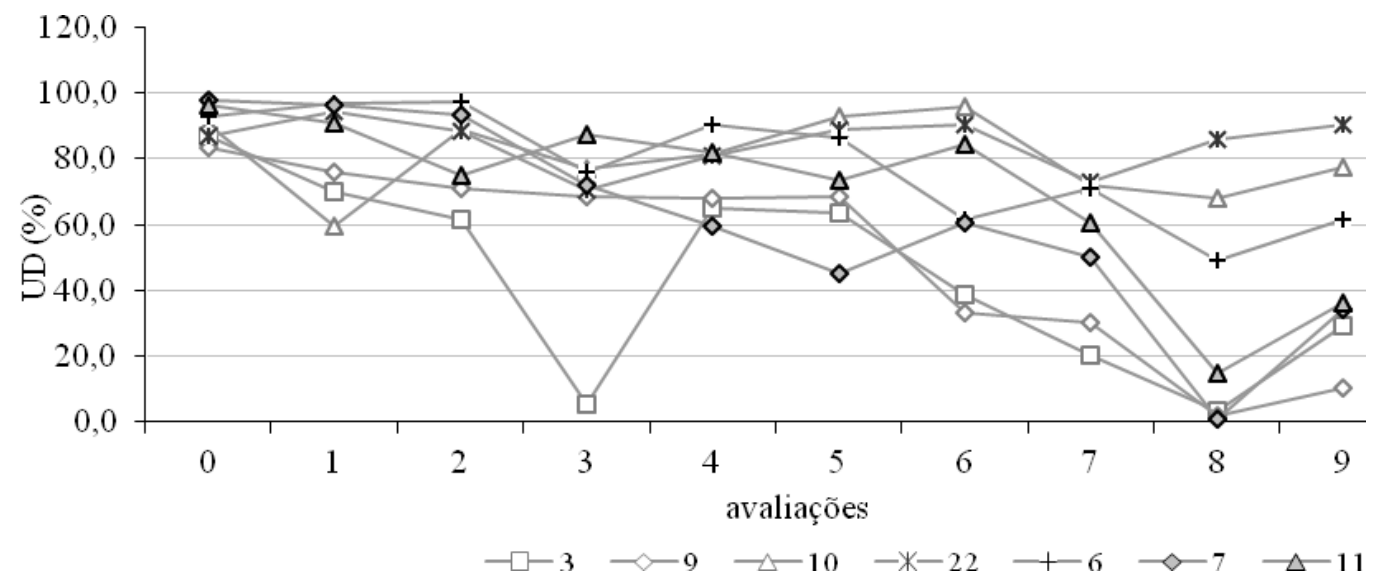

Rev. Bras. Agric. Irrigada v. 7, nº 1, p. 27 - 41 


\section{DESEMPENHO DE GOTEJADORES EM FUNÇÃO DA APLICAÇÃO DE TURFA GEL NA ÁGUA DE IRRIGAÇÃO}

Figura 8. Uniformidade de distribuição de água para os modelos de gotejadores do grupo 3.

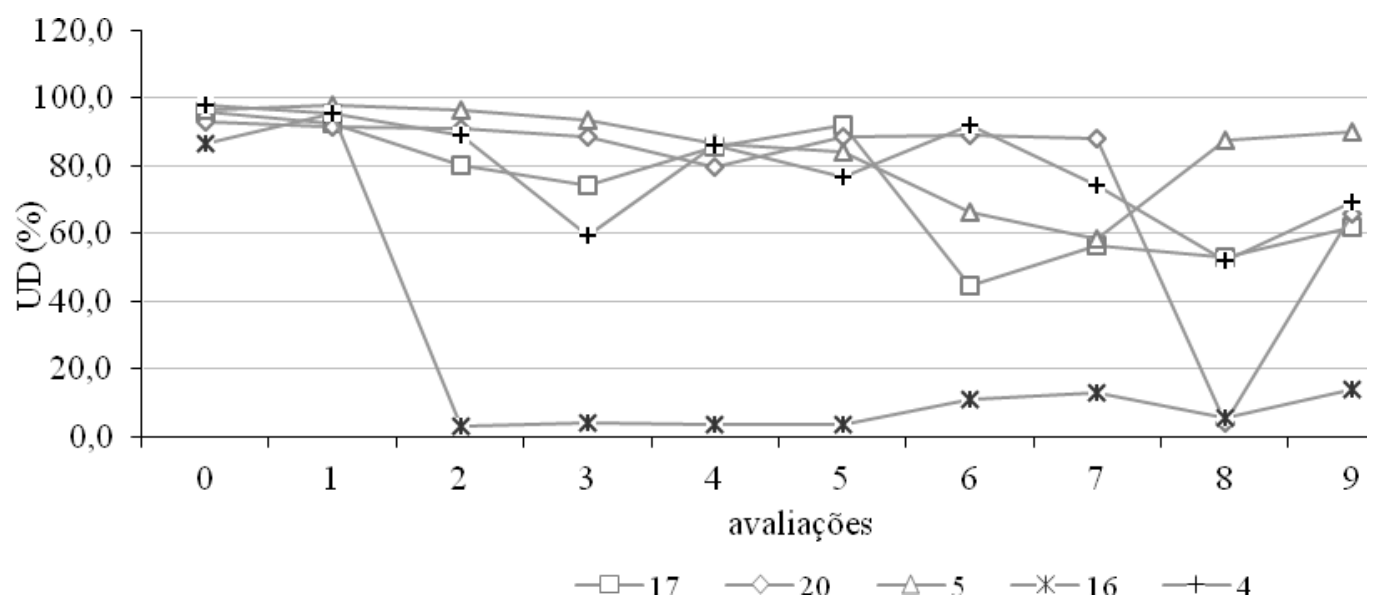

Figura 9. Uniformidade de distribuição de água para os modelos de gotejadores do grupo 4.

O modelo 25 (grupo 1) foi o único que apresentou uniformidade de distribuição de água próximo de $100 \%$, tendo ocorrido uma pequena alteração em seu comportamento quando a dosagem de turfa gel foi duplicada, recebendo assim, classificação aceitável (ASABE, 2003; SOLOMON, 1985). Os modelos 1, 21, 23, 24 e 26, apresentaram redução severa quanto à uniformidade de distribuição de água durante o ensaio.

$\mathrm{O}$ modelo 2 praticamente manteve os valores de UD acima de $80 \%$, caracterizando o melhor comportamento entre os modelos que compõem o grupo 2 . $\mathrm{O}$ modelo 12 apresentou redução drástica de UD nas últimas três aplicações de turfa gel, não apresentando, inclusive, nenhuma recuperação de vazão após a aplicação de tratamento químico na última avaliação (150 ppm de cloro livre + ácido fosfórico $85 \%$ P.A. e uso de "pigs" para limpeza da linha gotejadora a $300 \mathrm{kPa}$ ), mostrando ser altamente suscetível ao entupimento por turfa gel via água de irrigação.

Para o grupo 3, o modelo 22 manteve os valores de UD acima de $80 \%$, enquanto os modelos 6 e 10 apresentaram UD entre 60 e $80 \%$, e os demais modelos, valores abaixo de $60 \%$, recebendo classificação inaceitável conforme Solomon (1985).
O modelo 5 (grupo 4) apresentou redução da UD durante todo o ensaio, com exceção da avaliação número 8 que corresponde à última aplicação de turfa gel que contribuiu para o aumento da vazão seguido do tratamento químico para desobstrução utilizado na última avaliação, em que ocorreu uma pequena recuperação da vazão deste modelo de gotejador.

Quanto ao tratamento químico para recuperação de vazão, os gotejadores que apresentaram o melhor desempenho foram os modelos 15, 17 e 19, e o modelo 12 foi insensível ao tratamento químico para recuperação da vazão.

\section{CONCLUSÕES}

Os resultados obtidos na condução do ensaio permitiram chegar às seguintes conclusões:

a. A aplicação de turfa gel na concentração de dois litros para cada 300 litros de água agravou os problemas de entupimento, mostrando que para a correta utilização desse produto via água de irrigação deve ser utilizada a concentração mínima recomendada pelo fabricante a fim de evitar alterações quanto à uniformidade 


\section{DESEMPENHO DE GOTEJADORES EM FUNÇÃO DA APLICAÇÃO DE TURFA GEL NA ÁGUA DE IRRIGAÇÃO}

de aplicação de água dos diferentes modelos de gotejadores utilizados neste ensaio.

b. O modelo 25 (grupo 1) foi o único que apresentou uniformidade de distribuição de água próximo de $100 \%$ e baixo valor de coeficiente de variação de vazão durante todo o ensaio, recebendo assim, classificação aceitável (SOLOMON, 1985; ABREU et al. 1987).

c. O uso de tratamento químico à base de cloro $150 \mathrm{ppm}$ de cloro livre (hipoclorito de sódio) + ácido fosfórico $85 \%$ P.A. para manutenção do pH entre 5,5 e 6,0 + $300 \mathrm{kPa}$ associado ao uso de "pigs"

\section{REFERÊNCIAS BIBLIOGRÁFICAS}

ABREU, J. M. H.; LOPES, J. R.; REGALADO, A. P.; HERNANDEZ, J. F. G. El Riego localizado. Madrid: Instituto Nacional de Investigações Agrárias, 1987. $317 \mathrm{p}$.

ASABE EP 405. Design and installation of microirrigation systems.

ASAE

Standards, St. Joseph, p.900-905, 2003.

BARROS, A. C.; COELHO, R. D., MEDEIROS, P. R. F.; MELO, R. F. de; BARBOZA JÚNIOR, C. R. A.; DIAS, C. T. dos S. Entupimento de gotejadores em função da aplicação de superfosfato simples e ácido nítrico. Engenharia Agrícola, Jaboticabal, v.29, n.1, p.62-71, 2009.

BOMAN, B.J. Effects of orifice size on microsprinkler clogging rates. Transactions of the ASAE, St. Joseph, v.11, n.6, p.839-843, 1995.

GILBERT, R. G.; FORD, H. W. Operational principles/emitter clogging. In: NAKAYAMA, F.S.; BULKS, D.A. Trickle irrigation for crop production. (pedaço de esponja), contribuiu para a recuperação da vazão dos gotejadores em mais de $20 \%$, com exceção do modelo 12 , que foi insensível ao tratamento químico.

\section{AGRADECIMENTOS}

Os autores agradecem ao Ministério da Ciência e Tecnologia (MCT), ao Conselho Nacional de Desenvolvimento Científico e Tecnológico (CNPq) e à Coordenação de Aperfeiçoamento de Pessoal de Nível Superior (Capes), pelo apoio financeiro a esta pesquisa, através do Instituto Nacional de Ciência e Tecnologia em Engenharia da Irrigação (Inctei).

Amsterdam: Elsevier, 1986. cap.3. p.142163.

HANDRECK, K.; BLACK, N. Growing media for ornamental plants and turf. Sydney: University of New South Wales Press, 1999. 448p.

KELLER, J.; BLIESNER, D. R. Sprinkler and trickle irrigation. New York: von Nostrand Reinhold, 1990. 652p.

MELO, L. C. A.; SILVA, C. A; OLIVEIRA, D. B. Caracterização da matriz orgânica de resíduos de origens diversificadas. Revista Brasileira da Ciência do Solo, v.32, n.2, p.101-110, 2008.

PITTS, D.; PETERSON, K.; GILBERT, G.; FASTENAU, R. Field assessment of irrigation system performance. Transactions of the ASAE, St. Joseph, v.12, n.3, p.307-313, 1996.

RESENDE, R. S.; COELHO, R. D.; PIEDADE, S. M. de S. Suscetibilidade de gotejadores ao entupimento de causa biológica. Rev. bras. eng. agríc. 


\section{DESEMPENHO DE GOTEJADORES EM FUNÇÃO DA APLICAÇÃO DE TURFA GEL NA ÁGUA DE IRRIGAÇÃO}

ambiental, Campina Grande, v.4, n.3, p.368-375, 2000.

RIBEIRO, P. A. de A.; COELHO, R. D.; M. B. TEIXEIRA. Entupimento de tubos gotejadores convencionais com aplicação de cloreto de potássio (branco e vermelho) via duas qualidades de água. Engenharia Agrícola, Jaboticabal, v.30, n.2, p.279$287,2010$.

SAGI, G.; PAZ, E.; RAVINA, I; SCHISCHA, A; MARCU, A; YECHIELY, Z. Clogging of drip irrigation systems by colonial protozoa and sulfur bacteria. In. INTERNATIONAL

MICROIRRIGATION CONGRESS， 5, Orlando, 1995. Proceedings. St. Joseph: ASAE, 1995. p.250-254.

SCALOPPI, E.J. Características dos principais sistemas de irrigação. ITEMIrrigação e Tecnologia Moderna, Brasília, n.25, p.22-7, 1986.

SOLOMON, K. Global uniformity of trickle irrigation systems. Transactions of the ASAE, St. Joseph, v.28, n.4, p.1151$1158,1985$.
SOUSA, V. F. de; MAROUELLI, W. A.; COELHO, E. F.; PINTO, J. M.; COELHO FILHO, M. A. Irrigação e fertirrigação em fruteiras e hortaliças. Embrapa: Informação Tecnológica, Brasília, 2011, $771 \mathrm{p}$.

TEIXEIRA, M. B.; MELO, R. F. de; COELHO, R. D.; RETTORE NETO, O.; RIBEIRO, P. A. de A. Tratamento para desentupimento de gotejadores convencionais. Brazilian Journal of Irrigation and Drainage - IRRIGA, Botucatu, v.13, n.2, p.235-248, 2008. 